\title{
Disruption of the Centrifugal Visual System Inhibits Early Eye Growth in Chicks
}

\author{
Christopher Mark Dillingham, ${ }^{1}$ Jeremy Andrew Guggenheim, ${ }^{2}$ and Jonathan Thor Erichsen ${ }^{1}$ \\ ${ }^{1}$ School of Optometry and Vision Sciences, Cardiff University, Cardiff, Wales, United Kingdom \\ ${ }^{2}$ Centre for Myopia Research, Hong Kong Polytechnic University, Hung Hom, Kowloon, Hong Kong
}

Correspondence: Jonathan T. Erichsen, School of Optometry and Vision Sciences, Cardiff University, Maindy Road, Cardiff CF24 4HQ, Wales, UK; ErichsenJT@cf.ac.uk.

Submitted: December 21, 2012 Accepted: April 9, 2013

Citation: Dillingham CM, Guggenheim JA, Erichsen JT. Disruption of the centrifugal visual system inhibits early eye growth in chicks. Invest Ophthalmol Vis Sci. 2013;54:3632-3643. DOI:10.1167/iovs.12-11548
Purpose. Emmetropization, the process by which neonatal refractive errors are reduced toward zero, is partially dependent on brain-retina connectivity. Here, we investigated the role of the centrifugal visual system, a visually driven retinal feedback projection, as one potential influence on this complex mechanism.

Methods. Lesions of the isthmo-optic nucleus/tract or sham surgeries were performed in fiftyfour 4- to 5-day-old chicks to disrupt centrifugal efferents to the contralateral retina. Prior to surgery, baseline refractive error measurements were made using streak retinoscopy. Postoperative ocular phenotyping, which (in addition to retinoscopy) comprised A-scan ultrasonography and infrared keratometry, was performed 7 days and 21 days postsurgery. A pathway-tracing paradigm was used to determine lesion success, whereby an injection of wheat-germ agglutinin was made into the vitreous chamber contralateral to the lesion. Postmortem, tissue processing, immunohistochemistry, and stereological analysis of intact centrifugal neurons were performed. Subsequently, chicks were divided into quartile groups based on percentage lesion success.

REsults. Seven days postsurgery, chicks in the quartile of highest percentage lesion success exhibited significant axial hyperopia in the "treated eye" (contralateral to the lesion) relative to the "control eye" (ipsilateral to the lesion) eye, when compared with subjects within quartile groups of lower percentage lesion success $(P=0.004)$. However, by 21 days postsurgery, the induced hyperopia was no longer evident.

Conclusions. Unilateral disruption of centrifugal efferents to the retina of the contralateral eye induces an initial axial hyperopia, which is subsequently reversed through increased vitreous elongation in the affected eyes.

Keywords: isthmo-optic nucleus, electrolytic lesion, ectopic area
$\mathrm{T}_{\mathrm{i}}^{\mathrm{h}}$ he chick has been the most widely used model for investigations into the mechanisms of emmetropization, the term given to the process by which neonatal refractive error (REF) is reduced toward zero or low hyperopia. ${ }^{1,2}$ The consensus of numerous previous studies is that emmetropization is an active, visually guided process, which is in turn aided by genetically determined, nonvisually guided growth. ${ }^{3-5}$

A spectacle lens fitted in front of a chick's eye induces changes in the rate of ocular expansion to accurately negate the power of the lens. ${ }^{3,6}$ Thus, a positive lens would cue a reduction in the normal rate of globe expansion in combination with a thickening of the choroid until the combined optical power of the spectacle lens and anterior segment matched the focal plane, ${ }^{7,8}$ corresponding to the position of the retina. Once normal visual experience has been resumed (i.e., by removing the lens), the affected eye is able to recover, correcting for the induced hyperopic defocus. ${ }^{4}$ This recovery can be achieved only if the chick is still in the critical phase of development, which is characterized by the capability of a high ocular growth rate, relative to adulthood. 6,9

Importantly, when the retina is disconnected from the brain experimentally, whether through optic nerve section (ONS), ${ }^{10}$ exposure of retinal ganglion cells (RGCs) to tetrodotoxin (TTX) ${ }^{11}$ that blocks RGC action potentials, or colchicine, ${ }^{12}$ which selectively destroys RGCs, eyes retain the ability to respond to visual cues. ${ }^{4,13}$ Indeed, ONS eyes are still able to respond locally to defocus or form deprivation (FD) that is confined to local retinal regions. ${ }^{14,15}$ In addition to the above-mentioned surgical and chemical manipulations, the effects of ciliary nerve section (CNS) $)^{13}$ or lesion of the Edinger-Westphal nucleus have been investigated. ${ }^{16}$ These manipulations eliminate accommodation entirely or prevent only defocus-driven accommodation, respectively, and yet eyes retain the ability to respond to visual cues, such as lens manipulation or FD. Thus, it is generally recognized that active emmetropization is a mechanism largely driven by intraocular mechanisms. ${ }^{17}$ That being said, ONS studies in the chick have suggested that isolation of the retina from the brain may alter the refractive set point of the treated eye. ${ }^{3,4}$ Furthermore, following ONS but exposed to otherwise normal visual conditions, eyes develop moderate levels of hyperopia. ${ }^{4,10,13}$ Such findings argue that an eye-brain neural connection is necessary at least for fine-tuning emmetropization (Table 1). However, it is important to note that ONS surgery not only disrupts retinal ganglion cell efferents to primary visual centers of the brain, but also axons of the 
TABLE 1. The Effect of Lesion or Neurotoxin-Induced Effects on Emmetropization

\begin{tabular}{|c|c|c|c|c|}
\hline Treatment & Visual Manipulation & Treatment Effect & Reference & Contradictory Findings \\
\hline \multirow[t]{4}{*}{ ONS } & Normal VE & Hyperopia & 10,13 & 53 \\
\hline & & Reduced VCD & & \\
\hline & FD & Exaggerated axial length changes & 13,72 & \\
\hline & Minus lens & Reduced refractive compensation & 4 & \\
\hline CNS & Normal VE & Axial elongation & 13 & \\
\hline \multirow[t]{2}{*}{ ONS + CNS } & Normal VE & Axial elongation & 13 & \\
\hline & Minus lens & Over-compensation to minus lens power & 13 & \\
\hline \multirow[t]{3}{*}{ TTX } & Normal VE & Flatter cornea & 11 & 4 \\
\hline & & Reduced ACD & & \\
\hline & & Hyperopia & & \\
\hline \multirow{5}{*}{ Colchicine } & Normal VE & Myopia & 73 & 12 \\
\hline & & Axial elongation & 12,73 & \\
\hline & & Thinner lens & & \\
\hline & Minus lens & Reduced axial length compensation & 12 & \\
\hline & FD & Reduced axial length compensation & 73 & \\
\hline
\end{tabular}

Surgical or chemical disconnectivity of ocular components, such as the retina (ONS, colchicine, TTX) or the ciliary nerve (CNS), or combinations of these manipulations, such as optic nerve and ciliary nerve transection (ONS + CNS), disrupt compensation to experimental manipulations of visual experience.

midbrain centrifugal visual system that terminate on isthmooptic target cells (IOTCs) in the ventral retina.

The centrifugal visual system (CVS), elements of which (i.e., retinopetal neurons) are found in many vertebrates, ${ }^{18}$ is defined by an efferent projection from the brain to the retina, one that is particularly enlarged and well defined in birds ${ }^{19}$ (for a recent review, see Wilson and Lindstrom ${ }^{20}$ ). As such, the avian class has been the focus of the majority of the functional, anatomic, and physiologic studies of the CVS, such as in the chicken, ${ }^{21-23}$ pigeon, ${ }^{24-26}$ and Japanese quail. $^{27,28}$ In the chicken and pigeon, 10,000 to 12,000 neurons, respectively, project in a highly topographic manner from layers 9 and 10 of the optic tectum, ${ }^{29-31}$ a structure analogous to the mammalian superior colliculus. This pathway (the tecto-isthmic tract) transmits predominantly, but not exclusively, visual information to the isthmooptic nucleus (ION) (and potentially neurons of the surrounding ectopic area [EA]). In turn, both of these midbrain centrifugal neuronal populations have major projections to the contralateral retina via the isthmo-optic tract (IOTr), ${ }^{32}$ whereas a small subpopulation of ectopic neurons project to the ipsilateral retina ${ }^{33}$ via an as yet undefined pathway. Within the retina, so-called restricted efferent centrifugal fibers, which arise from isthmo-optic neurons, terminate on single IOTCs in a distribution confined to the ventral retina. ${ }^{34}$ These so-called divergent efferent axons, ${ }^{35}$ from centrifugal ectopic neurons, terminate on numerous target cells within the retina (including displaced retinal ganglion cells, the primary afferent input to the accessory optic system ${ }^{36}$ ), again in a distribution confined to the ventral retina. Secondary axons arising from IOTCs innervate RGCs in a seemingly random distribution that covers the entire retina. ${ }^{37}$ Neurochemical studies of these IOTCs reveal nitric oxide synthase (NOS) activity, ${ }^{38}$ a finding of potential significance as treatment of the retina with the NOS-inhibitor, L-NAME, prevents FD-induced myopia. ${ }^{39}$ In addition, L-NAME prevents choroidal thinning in response to occluder wear, and thickening after occluder removal. ${ }^{40}$ The aim of the present study was to elucidate the effect of unilateral disruption of the projections from ION and surrounding EA to the contralateral retina on the capability for, or accuracy of, emmetropization under otherwise normal developmental conditions.

\section{MeTHODS}

\section{Animals and Treatments}

A total of 54 Shaver Black chicks (a cross between Rhode Island Red and Barrack Rock strains), obtained as fertilized eggs from a hatchery specializing in biomedical research (Henry Stewart Ltd., London, UK), were used in this study. Once hatched, the chicks were transferred to a custom-made, temperature-controlled $\left(25-27^{\circ} \mathrm{C}\right)$ brooding pen for the first week, before being moved to a larger pen heated by an overhead lamp for the remainder of the study. Food and water were available without restriction, with the exception of 1 to 2 hours prior to induction of general anesthesia. During this brief period, chicks were not provided with food but had unlimited access to water. Noncycloplegic streak retinoscopy was performed on awake chicks 3 days posthatch to ensure that no unusual ametropia was evident in either eye prior to treatment (prescreen). Four to 5 days posthatch (37-45 g body weight), general anesthesia was induced (and maintained as necessary) by intramuscular injection of ketamine hydrochloride $(50 \mathrm{mg} / \mathrm{kg})$ and xylazine $(3.5 \mathrm{mg} / \mathrm{kg})$, combined with a separate preoperative intramuscular injection of analgesic (ketoprofen, $0.1 \mathrm{mg} / \mathrm{kg}$ ). Once deeply anesthetized (as determined primarily by lowered respiratory rate and an absent toe-pinch reflex), subjects were placed in a stereotaxic frame (David Kopf Instruments, Tujunga, CA). Initially, the scalp was incised to reveal the dorsocaudal cranium. A craniotomy was performed using a dental drill, revealing the dura mater. A small cut was made in the dura using a microscalpel, and a tungsten electrode, insulated except for the tip (Pan AM Systems, Portsmouth, NH), was lowered to coordinates, predetermined using the stereotaxic head position of Kuenzel and Masson, ${ }^{41}$ corresponding either to the left ION (anteroposterior [AP]; +2.014 , mediolateral $[\mathrm{ML}] ;-1.750$, dorsoventral [DV]; +4.546) or the left IOTr (AP; +2.354, ML; -2.035 , DV; 5.125) before being lowered a further $100 \mu \mathrm{m}$ for 3 to 4 minutes to penetrate the pia mater overlying the dorsal midbrain. The electrode was then raised back to the previous dorsoventral position. An electrolytic lesion was made by passing $0.90-\mathrm{mA}$ DC current for 15 to 20 seconds. The electrode was left in place for a further 15 seconds before being disconnected from the stimulus isolator and raised out of the brain. Instead of receiving an ION or IOTr lesion, a proportion of subjects underwent a sham procedure whereby 
lesions were placed in brain regions proximal to the IOTr, but that did not disrupt centrifugal efferents $(n=6)$. The craniotomy was filled using bone wax (if required) and the scalp incision sutured. A custom-made hard-plastic concave disc was glued with cyanoacrylate adhesive to feathers surrounding the wound so that unwanted pecking from brooder mates did not compromise the healing process.

\section{Light Conditions}

The illumination in the brooder and floor pen was 250 to 300 lux. Subjects were raised under diurnal conditions ( $14 \mathrm{~h}$ light/ 10 h dark cycle).

\section{Measurements}

For a detailed methodology of the ocular phenotyping techniques used, refer to Prashar et al. ${ }^{42}$ Prior to surgery, REF and body weight (to the nearest gram) measurements were taken (prescreen). After a recovery period of 7 and 21 days postsurgery (PS), REF and corneal radius of curvature (CR) were measured on fully awake chicks using noncyclopleged streak retinoscopy and infrared photo keratometry, respectively. For the latter, a modified videokeratometer, based on the design of Schaeffel and Howland, ${ }^{43}$ was used. Highfrequency A-scan ultrasonography was performed on anesthetized chicks at 7 and 21 days PS to measure ocular component dimensions: ACD, anterior chamber depth; LT, lens thickness; VCD, vitreous chamber depth; and AXL, axial length. Body weight was also measured to the nearest gram at 7 days and at 21 days PS. All measurements were taken by an observer masked to the treatment group (i.e., lesion or sham operated), and lesion success, which was established postmortem.

\section{Determination of Lesion Success}

Damage to the IOTr caused by the electrolytic lesion was intended to disrupt the centrifugal efferents to the contralateral retina. An intravitreal injection of unconjugated wheatgerm agglutinin (WGA, L-1020; Vector Laboratories Ltd., Peterborough, UK) was administered in the contralateral eye 48 hours prior to perfusion, to label all residual ION and EA neurons. The degree of induced disruption could then be assessed quantitatively through a comparison of the number of retrogradely labeled CVS neurons in lesioned animals with the mean number of retrogradely labeled centrifugal neurons in the intact CVS of sham-operated subjects. Using this method, the degree of success of each lesion was expressed as the percentage of centrifugal neurons that were destroyed.

Specifically, after the ultrasonography measurement at 21 days PS, but while the birds were still under anesthesia, $25 \mu \mathrm{L}$ of WGA $(10 \mathrm{mg} / \mathrm{mL})$ was injected into the vitreous chamber of the eye contralateral to the hemisphere in which the IOTr/ION lesion had been made or the sham surgery performed. The eyelid was opened using custom-made lid retractors, and a pilot hole was made in the eye proximal to the scleral insertion of the superior rectus, using a 25 -gauge needle. A $50 \mu \mathrm{L}$ syringe (Hamilton Medical AG, Bonaduz, Switzerland) was used to inject $30 \mu \mathrm{L}$ of $1 \%$ WGA (w/v aq.) close to, but without damaging, the retina. The syringe was held in place for 1 to 2 minutes, to allow dispersion of the lectin within the globe, before being withdrawn. The eyelid was allowed to close naturally, and the chick's recovery from the anesthetic was monitored in a temperature-controlled recovery chamber. Once animals had regained alertness, balance, and the ability to perch (usually 45-60 minutes), they were returned to their pen. After a survival time of 48 hours postinjection, subjects were given an overdose of pentobarbital (Euthatal; Merial
Animal Health Ltd., Essex, UK) and perfused intracardially with appromately $300 \mathrm{~mL}$ of $0.1 \mathrm{M}$ phosphate buffered saline (PBS, $\mathrm{pH}$ : 7.35) followed by approximately $500 \mathrm{~mL}$ of $2.5 \%$ paraformaldehyde in $0.1 \mathrm{M}$ PBS. Brains were then excised and postfixed in the same solution for 48 hours and subsequently cryoprotected in a $20 \%$ sucrose/0.1 M phosphate buffer solution for a further 48 hours. Brains were then sectioned on a frozen-stage microtome (Micron Technology, Inc., Boise, ID) at $50 \mu \mathrm{m}$ in the coronal plane. Serial sections of each brain were collected in a " 1 in 3 " series. Every third section was mounted directly onto double gelatin-subbed slides for cresyl violet staining. These cresyl violet sections were used to determine the precise location of the lesion site (i.e., ION or IOTr; Fig. 1).

\section{Immunohistochemistry}

The remaining sections were collected in $0.1 \mathrm{M}$ Tris-buffered saline (TBS, pH 7.35). Of these latter sections, the second section of every third one cut was processed for $\alpha$-WGA immunohistochemistry, whereas the remaining sections were kept as backup tissue. After pretreatment with a solution of $0.3 \% \mathrm{H}_{2} \mathrm{O}_{2}$ and $10 \%$ methanol to remove endogenous peroxidases, free-floating sections were incubated at room temperature in a $3 \%$ solution of normal horse serum (NHS) (S2000; Vector Laboratories) diluted in 0.1 M Tris-buffered saline containing $0.005 \%$ Triton X-100 (TXTBS). Sections were then directly transferred to a solution containing a 1:1000 dilution of biotinylated anti-WGA (BA-0024; Vector Laboratories) in TXTBS with $1 \%$ NHS, where they were incubated for 24 hours at room temperature under continuous gentle agitation. After three intervening washes in TBS, sections were incubated under the same conditions in a 1:500 dilution of biotinylated rabbit-anti goat IgG (PK-6105; Vector Laboratories) in TBS with 1\% NHS. Again, after three intervening washes in TBS, the sections were incubated in a peroxidase complex (PK-6105; Elite ABC complex Vector Laboratories) (1:100) for 2 hours under the same conditions. Further washes in TBS preceded overnight acclimatization to $0.05 \mathrm{M}$ Tris buffer (TNS) at $4^{\circ} \mathrm{C}$. The resulting peroxidase activity was visualized by placing the sections in a $0.5 \%$ DAB solution diluted in TNS with $0.09 \%$ $\mathrm{H}_{2} \mathrm{O}_{2}$ until the color had developed sufficiently. After washes in TNS, sections were mounted on double gelatin-subbed slides, air-dried for 48 hours, dehydrated in ascending alcohols, cleared in xylene, and coverslipped in DPX mountant.

\section{Data Analysis}

A "1 in 3" series of $\alpha$-WGA labeled tissue, from each subject, was traced and neuronal counts were made (Model DM6000B Leica microscope, Leica Microsystems GmbH, Wetzlar, Germany, in conjunction with StereoInvestigator Software, Version 8; MBF Bioscience, Edinburgh, UK). Cell counts for contralaterally projecting ION and EA centrifugal neurons were made separately and then subtracted from the mean cell counts of ION and EA populations from sham-operated subjects, respectively. The relative loss in ION and EA cell number was then expressed as a percentage of centrifugal neuron/axon disruption. In analyzing the effects of lesions, subjects were ranked and split into three sets of independent quartile groups (Q1 to Q4) based on (1) ION lesion success $\left(I O N_{Q 1-4}\right)$, (2) EA lesion success $\left(E A_{Q 1-4}\right)$, and (3) ION and EA combined lesion

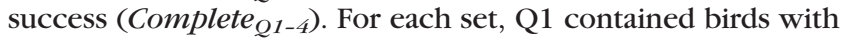
the lowest percentage lesion success (including sham-operated controls) and Q4 the highest. Ocular component measurements were analyzed as follows. For REF, the mean was taken of the two principal meridians for each eye. For A-scan ultrasonography, the mean was taken of six repeated measure- 


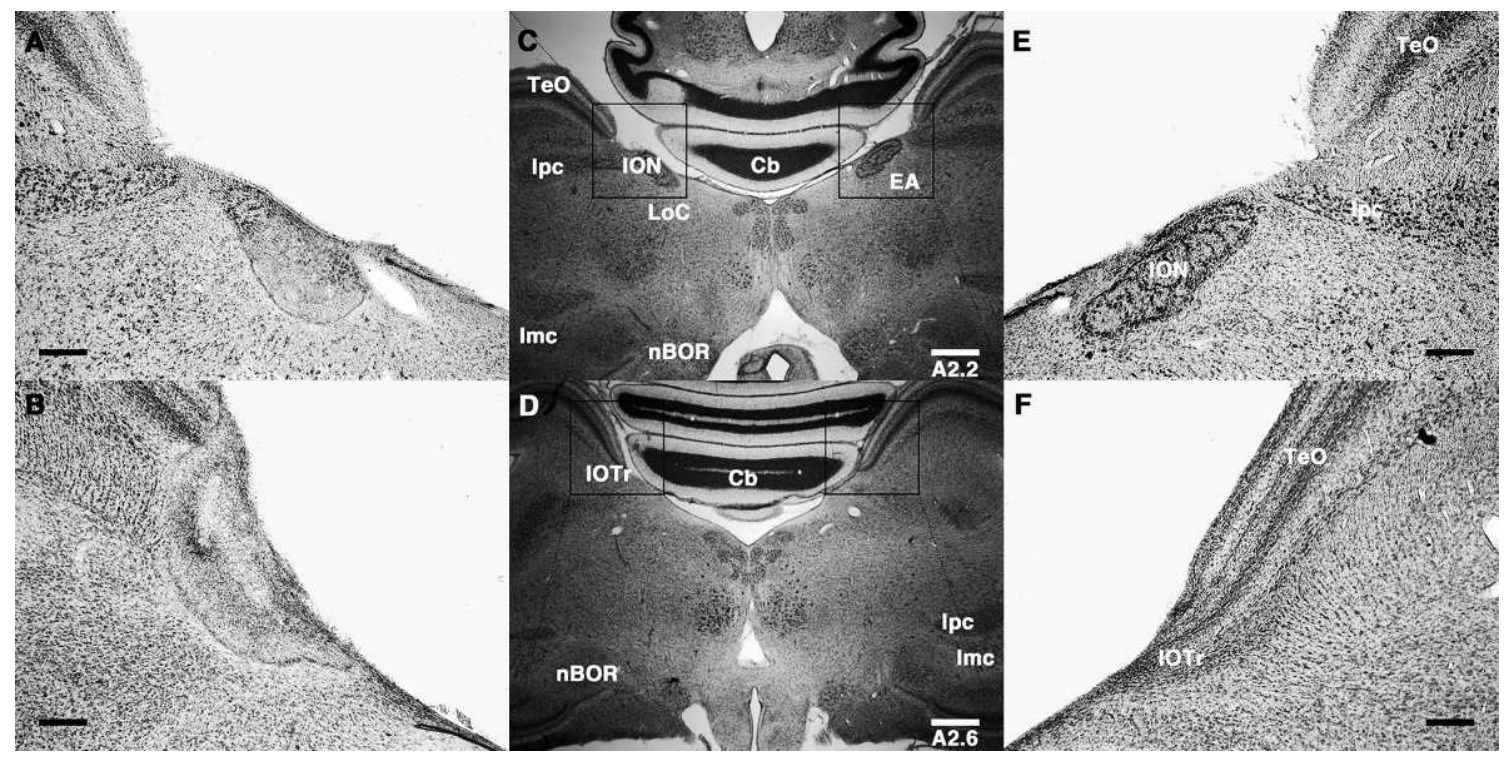

Figure 1. Examples of IOTr and ION lesion histology in cresyl violet-stained sections. The isthmo-optic tract (IOTr) courses rostrally from the isthmo-optic nucleus (ION) at the dorsomedial aspect of the optic tectum before merging with the optic tract. In these coronal sections of the chick midbrain (AP coordinates approximated according to the atlas of Kuenzel and Masson ${ }^{41}$ ), electrolytic lesions of the IOTr were made unilaterally, consistently on the left side of the midbrain, whereas the right side of the midbrain was left untreated. Low-magnification $(\times 125)$ photomicrographs of coronal sections through the chicken midbrain are shown, illustrating the target of ION (C) and IOTr lesions (D). A partially lesioned (A) and intact ION (E) and a lesioned (B) and intact an $\operatorname{IOTr}(\mathbf{F})$ are shown at higher magnification $(\times 500)$. Cb, cerebellum; EA, ectopic area; Imc, nucleus isthmi magnocellularis; LoC, locus ceruleus; Ipc, nucleus isthmi parvocellularis; nBOR, nucleus of the basal optic root; TeO, optic tectum. Scale bars $(\mathbf{C}, \mathbf{D}): 1000 \mu \mathrm{m} ;(\mathbf{A}, \mathbf{B}, \mathbf{E}, \mathbf{F}): 250 \mu \mathrm{m}$.

ments obtained from each eye. For corneal radius of curvature (CR), the mean was taken of six repeated measurements for each eye, with these measurements being derived from the average value along the two principal meridians

The mean within-animal (i.e., interocular difference for each ocular component measured) was calculated by subtraction of the control eye measurements from treated eye measurements (T-C). In all subjects, the treated eye (i.e., contralateral to the lesion of ION/IOTr) was the right eye and the control eye (i.e., ipsilateral to the lesion) was the left eye. All graphical and statistical analyses were carried out using the statistical software R (Version 2.15.2; provided in the public domain by the R Foundation for Statistical Computing, Vienna, Austria; R Development Core Team, 2009, available at http://www. r-project.org/). Within-animal asymmetry in refractive error (i.e., anisometropia) and ocular component dimension asymmetry (i.e., $\triangle A C D, \triangle L T, \triangle V C D, \triangle A X L$, and $\triangle C R$ ) were assessed using dependent sample $t$-tests. For statistical modeling, the CRAN package: "Ime4" was used (Bates and Maechler, 2010; http://cran.r-project.org/web/packages/lme4), whereas all graphs were generated using the "ggplot2" package (Wickham and Chang, 2012; http://cran.R-project.org/web/ packages/ggplot2).

A primary analysis of lesion effect on ocular measurements was performed using the Complete $_{Q 1-4}$ independent variable. Generalized linear mixed models (GLMMs) were generated by stepwise addition of the categorical fixed effects, Complete ${ }_{Q 1-4}$, Lesion site (i.e., IOTr or ION only), Days post surgery (Days PS), the interaction term "Complete ${ }_{O 1-4} \times$ Days PS," and the continuous fixed effects: Body weight $(g)$ and Prescreen anisometropia $(D)$, to investigate the direction and magnitude of associations with each dependent variable (i.e., the difference in measured ocular components [T-C], e.g., $\triangle A C D$ $[\mathrm{mm}])$. Model selection was based on the Akaike information criterion (AIC), a measure based on the explained deviance and the remaining degrees of freedom $(d f)$ of a given model and direct comparison of models prior to, and following addition of, a given fixed effect. ${ }^{44}$ Prescreen anisometropia measurements were included for the dependent variable, Anisometropia, only (note that baseline measurements for ocular component dimensions were not taken, and thus could not be included in models in an analogous manner to the above). A natural log link function and Gaussian distribution were specified for the dependent variable Anisometropia $(+2 \mathrm{D})$ so that the model's assumption of a normal distribution of residual variance was not violated. In all other cases, an integer link function (i.e., the natural form of the data) and a Gaussian family were specified.

The differential contribution of ION and EA damage to postsurgery changes in ocular component measurements was assessed through a comparison of the regression coefficients (B) and standardized effect sizes (b) of $I_{O N_{Q 1-4}}$ and $E A_{Q 1-4}$, that is, the strength of the relationship between parallel GLMMs containing either $I O N_{Q 1-4}$ or $E A_{Q 1-4}$ as fixed effect variables within a best-fit model, which had been determined a priori for each dependent variable. In addition, the relative contribution of either $I O N_{Q 1-4}$ or $E A_{Q 1-4}$ to the goodness of fit of the model was assessed through a direct comparison between a model containing either $I O N_{Q 1-4}$ or $E A_{Q 1-4}$, with a baseline model containing only the random effect, Subject ID, and an intercept of 1 .

To assess the independent contribution of treated and control eye changes to potential lesion-induced ocular component asymmetry, Spearman's Rho $(\rho)$ was calculated to assess the magnitude and direction of the association between the refractive error of each eye when plotted against percentage lesion success on a continuous linear scale. Shamoperated subjects were included in these correlation analyses based on cell counts of retrograde WGA labeling. As a result of interanimal variability in the number of retrogradely labeled centrifugal neurons, sham subjects with fewer than average centrifugal neurons were represented in the analysis by a corresponding percentage lesion success value. As such, sham subjects are all represented within a $0 \%$ to $20 \%$ range of lesion success. Data are presented as means \pm SD unless otherwise 
stated, whereas error bars on graphs represent $95 \%$ confidence intervals. A value of $P<0.05$ was considered statistically significant. Note that values of $P$ relating to GLMM analyses are the product of likelihood ratio tests, that is, the isolation of the significance of a fixed effect based on a comparison of nested models.

All experimental procedures involving animals complied with the applicable U.K. Home Office legislation and the European Communities Council Directive 86/609/EEC (1986) and were performed in accordance with the ARVO Statement for the Use of Animals in Ophthalmic and Visual Research.

\section{Results}

\section{Lesion Effect}

Lesions of the isthmo-optic tract (IOTr), isthmo-optic nucleus (ION) (Fig. 1), or those of the IOTr that were sufficiently caudal to affect ION as well (Fig. 1B), all disrupted centrifugal efferents that targeted the retina. Retrograde labeling of ION/ EA somata following intravitreal injection of WGA in the eye contralateral to the side of the lesion was reduced relative to sham-operated subjects irrespective of the lesion target. Although lesions of the IOTr effectively reduced retrograde labeling of centrifugal neurons, in no cases were these lesions $100 \%$ successful (i.e., some retrogradely labeled centrifugal cells were always observed).

Lesions of the ION/EA did achieve effectively $100 \%$ success (Fig. 2), and are reported as such when expressed as whole integers. However, even in these cases, a few $(<10)$ remaining cells were labeled. Although lesions of the ION were generally more effective, larger lesions were required, thus increasing the potential for damage to surrounding regions as well as more extensive effects from damage to vasculature (i.e., ischemic effects). Lesions that targeted the ION, when successful, in all likelihood also disrupted axon bundle formation at the rostral apex, caudal to the coalescence of fiber bundles that in turn form the IOTr. Thus, the distinction between the loci of lesions was not absolute. The optimum tradeoff between percentage lesion success and lesion size was achieved with those lesions that targeted the IOTr proximal to the ION, whereby the caudal extension of the lesion site affected the rostral apex of the nucleus and, thus, the fibers of the presumptive IOTr.

\section{Refractive Error}

Seven days PS, chicks in the Q4 group, as defined by the combined percentage lesion success of ION and EA cell counts (i.e., Complete ${ }_{Q 1-4}$ ), showed a highly significant mean hyperopic shift relative to control (ipsilateral) eyes $(2.30 \pm 2.46$ diopters [D], $d f=13, t=-3.50, P=0.004)$. No significant relative hyperopia was found in Q1 to Q3 groups of chicks, that is, sham-operated controls and those subjects with lower lesion success $(\sim 0-80 \%)$ (Table 2$)$. At 21 days PS, the anisometropia previously observed in the Q4 group of chicks was no longer apparent $(-0.17 \pm 0.48 \mathrm{D}, d f=13, t=1.31, P=$ 0.214). The Q2 and Q3 groups of chicks continued to show no significant anisometropia (Table 2). Unexpectedly, Q1-treated eyes showed a very small but statistically significant hyperopia relative to fellow control eyes $(0.17 \pm 0.27 \mathrm{D}, d f=13, t=$ $-2.38, P=0.033$ ) (Table 2).

To show the effect of IOTr/ION lesions on anisometropia, a best-fit model containing the categorical fixed effects, Complete $_{Q 1-4}$ and Days PS (7 or 21 days PS), as well as the interaction term, Complete C1-4 $_{1} \times$ Days PS, and Subject ID as a random term was generated. In the final, best-fitting model, the
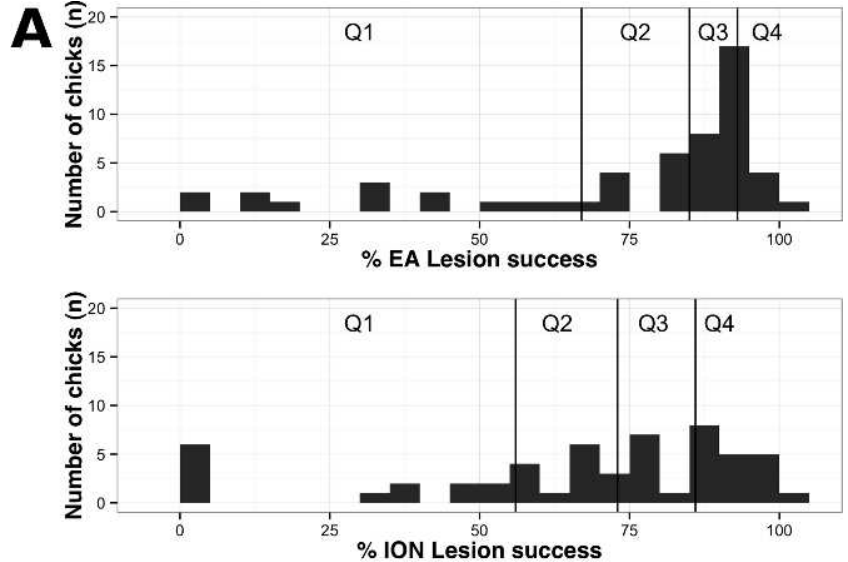

B

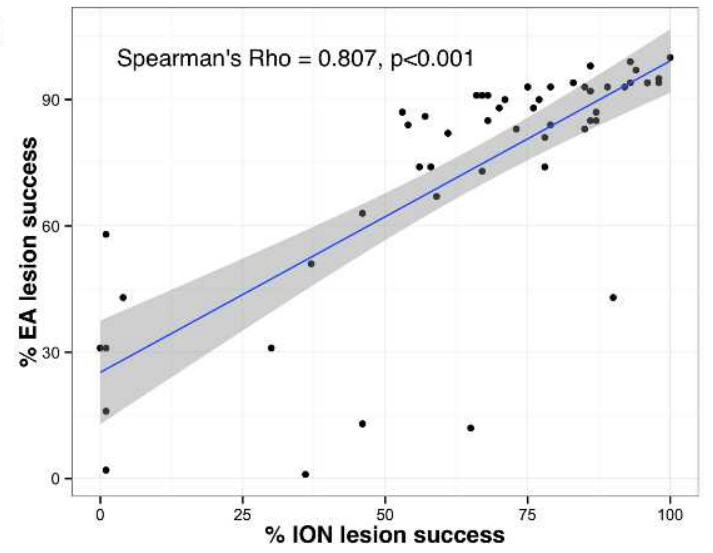

FIGURE 2. (A) Distribution of EA and ION percentage lesion success of individual subjects $(n=54)$ into quartile groups $(\mathrm{Q} 1-\mathrm{Q} 4)$. (B) A strong positive correlation existed between percentage ION and EA lesion success in chicks following lesion of the isthmo-optic tract (IOTr; $n=$ 24 ) or the isthmo-optic nucleus (ION; $n=20$ ), both IOTr and ION ( $n=$ 4) or sham-operated controls $(n=6)$. The shaded region around the trend line in (B) represents $95 \%$ confidence intervals.

Q4 group of chicks exhibited a strong positive relationship with the level of anisometropia over the duration of the measurement period $(B=0.786 \pm 0.137, t=5.758)$, whereas the regression coefficients of the quartile groups with lower lesion success (i.e., Q3-Q1) were consistently weaker (Table 3).

The interaction term containing the fixed effects Complete $e_{Q 1-4} \times$ Days $P S$, significantly improved the fit of the model $\left(\chi^{2}=25.716, d f=3, P<0.001\right)$. Indeed, a strong negative relationship was evident in the effect of Complete C1-4 $_{Q 1-4}$ over Days PS $(B=-1.041 \pm 0.195, t=-5.351)$. Thus, as one would expect given the transient nature of the observed anisometropia over time (Fig. 3A), the strength of the effect of Qcomplete $_{Q 1-4}$ was significantly different at the two measurement time points, as defined by the Days PS term (Table 2).

\section{Ocular Component Dimensions}

Seven days PS, the treated eyes of the Q4 group of chicks had significantly shorter VCD $(-0.123 \pm 0.142 \mu \mathrm{m}, d f=13, t=$ $-3.246, P=0.006)$ and AXL $(-0.121 \pm 0.141 \mu \mathrm{m}, d f=13, t=$ $-3.216, P=0.007$ ) than fellow control eyes. Small but statistically significant VCD $(0.024 \pm 0.031 \mu \mathrm{m}, d f=13, t=$ $2.905, P=0.012)$ and AXL $(0.033 \pm 0.026 \mu \mathrm{m}, d f=13, t=$ $4.704, P<0.001)$ asymmetry between the two eyes was also observed in the Q1 group of chicks. No significant within- 
TABLE 2. Ocular Component Measurement Asymmetry (Mean \pm SD; T-C) Across Quartile Groups of Combined ION and EA Lesion Success at 7 and 21 DPS

\begin{tabular}{|c|c|c|c|c|}
\hline & \multicolumn{4}{|c|}{ Ocular Component Asymmetry, T-C } \\
\hline & Q1, $n=14$ & $\mathbf{Q} 2, n=13$ & Q3, $n=13$ & Q4, $n=14$ \\
\hline \multicolumn{5}{|l|}{$7 \mathrm{~d}$ PS } \\
\hline Anisometropia (D) & $0.05 \pm 0.36$ & $0.20 \pm 0.96$ & $0.42 \pm 1.21$ & $2.31 \pm 2.46 \neq$ \\
\hline$\triangle A C D, \mathrm{~mm}$ & $0.003 \pm 0.014$ & $0.002 \pm 0.016$ & $0.000 \pm 0.017$ & $0.005 \pm 0.024$ \\
\hline$\Delta L T, \mathrm{~mm}$ & $0.006 \pm 0.022$ & $-0.003 \pm 0.023$ & $-0.005 \pm 0.022$ & $-0.003 \pm 0.018$ \\
\hline$\Delta V C D, \mathrm{~mm}$ & $0.024 \pm 0.031^{*}$ & $0.007 \pm 0.070$ & $-0.010 \pm 0.061$ & $-0.123 \pm 0.142 \dagger$ \\
\hline$\triangle A X L, \mathrm{~mm}$ & $0.033 \pm 0.026 \ddagger$ & $0.006 \pm 0.070$ & $-0.015 \pm 0.063$ & $-0.121 \pm 0.141 \dagger$ \\
\hline$\Delta C R, \mathrm{~mm}$ & $0.004 \pm 0.049$ & $-0.007 \pm 0.050$ & $-0.010 \pm 0.039$ & $0.029 \pm 0.061$ \\
\hline \multicolumn{5}{|l|}{$21 \mathrm{~d}$ PS } \\
\hline Anisometropia (D) & $0.17 \pm 0.27^{*}$ & $0.03 \pm 0.24$ & $-0.05 \pm 0.34$ & $-0.17 \pm 0.48$ \\
\hline$\triangle A C D, \mathrm{~mm}$ & $-0.000 \pm 0.017$ & $-0.001 \pm 0.015$ & $0.001 \pm 0.022$ & $-0.012 \pm 0.025$ \\
\hline$\Delta L T, \mathrm{~mm}$ & $0.010 \pm 0.023$ & $0.006 \pm 0.019$ & $0.007 \pm 0.026$ & $0.008 \pm 0.023$ \\
\hline$\triangle V C D, \mathrm{~mm}$ & $0.012 \pm 0.050$ & $0.029 \pm 0.043^{*}$ & $0.029 \pm 0.035^{*}$ & $0.038 \pm 0.059^{*}$ \\
\hline$\triangle A X L, \mathrm{~mm}$ & $0.022 \pm 0.048$ & $0.034 \pm 0.047^{*}$ & $0.036 \pm 0.034 \ddagger$ & $0.033 \pm 0.062$ \\
\hline$\Delta C R, \mathrm{~mm}$ & $0.031 \pm 0.058$ & $0.034 \pm 0.071$ & $0.035 \pm 0.057^{*}$ & $0.045 \pm 0.065^{*}$ \\
\hline
\end{tabular}

Statistical test results (dependent $t$-tests) relate to within-group analyses (i.e., is treated eye different from control eye?). Between-group comparisons are shown in Figures 2 (Anisometropia) and 3 (ocular component dimensions).

$$
\begin{aligned}
& * P<0.05 . \\
& +P<0.01 . \\
& \ddagger P<0.005 .
\end{aligned}
$$

subject difference was found in either the ACD or LT of any quartile groups. Similarly, in GLMM analyses, no significant relationships were observed between any of the independent variables (Complete ${ }_{Q 1-4}$, Body weight, Days PS, Lesion site) and either the $\triangle A C D$ or $\triangle L T$ (Table 3). Twenty-one days PS, no significant difference was found between treated and fellow control eyes in either ACD or LT components. The VCD and AXL of treated eyes in the Q2 to Q4 groups of chicks were statistically significantly longer when compared with fellow control eyes (Table 2); however, the differences were marginal when compared with those observed at 7 days PS. In fact, the consistent magnitude of the small, latter asymmetry could be attributed to a systematic measurement artifact, the implications of which are discussed in the following text.

As one would expect, given the strong inverse relationship of Anisometropia with both $\triangle V C D(\rho=-0.641, P<0.001$; Fig. 3B) and therefore $\triangle A X L(\rho=-0.601, P<0.001)$, the best-fit model structure for both the $\triangle V C D$ and $\triangle A X L$ dependent variables mirrored that of the dependent variable, Anisometropia. In the case of both $\triangle V C D$ and $\triangle A X L$, the Q4 group of chicks exhibited a strong negative relationship with both $\triangle V C D(B=-0.147 \pm 0.027, b=-0.123, t=-5.541)$ and $\triangle A X L$ $(B=-0.154 \pm 0.027, b=-0.121, t=-5.785)$. A strong positive association between the interaction term, Qcomplete $_{Q 1-4} \times$ Days $P S$, and both $\triangle V C D(B=0.165 \pm 0.032, t=5.205)$ and

\begin{tabular}{|c|c|c|c|c|c|c|c|}
\hline \multirow{2}{*}{$\begin{array}{c}\text { Dependent } \\
\text { Variable }\end{array}$} & \multicolumn{2}{|c|}{ Predictors } & \multirow[b]{2}{*}{$\boldsymbol{B}$} & \multirow[b]{2}{*}{$\boldsymbol{b}$} & \multirow[b]{2}{*}{$t$} & \multicolumn{2}{|c|}{$\Lambda$} \\
\hline & Cell Groups & Quartile Group & & & & $\chi^{2}$ & $\boldsymbol{P}$ \\
\hline \multirow[t]{8}{*}{ Anisometropia, D } & $\mathrm{ION}_{\mathrm{Q} 1-4}$ & Q1 & 0.018 & 0.018 & 0.068 & 19.898 & 0.0002 \\
\hline & & Q2 & 0.136 & 0.154 & 0.357 & & \\
\hline & & Q3 & 0.194 & 0.212 & 0.508 & & \\
\hline & & Q4 & 2.554 & 2.571 & 6.832 & & \\
\hline & $\mathrm{EA}_{\mathrm{Q} 1-4}$ & Q1 & 0.033 & 0.033 & 0.121 & 14.516 & 0.0023 \\
\hline & & $\mathrm{Q} 2$ & 0.071 & 0.104 & 0.172 & & \\
\hline & & Q3 & 0.505 & 0.538 & 1.254 & & \\
\hline & & Q4 & 2.270 & 2.304 & 5.746 & & \\
\hline \multirow[t]{8}{*}{$\Delta V C D, \mathrm{~mm}$} & $\mathrm{ION}_{\mathrm{Q} 1-4}$ & Q1 & 0.022 & 0.022 & 1.201 & 15.999 & 0.0011 \\
\hline & & $\mathrm{Q} 2$ & -0.018 & 0.004 & -0.695 & & \\
\hline & & Q3 & -0.019 & 0.002 & -0.748 & & \\
\hline & & Q4 & -0.151 & -0.129 & -5.827 & & \\
\hline & $\mathrm{EA}_{\mathrm{Q} 1-4}$ & Q1 & 0.025 & 0.025 & 1.704 & 10.755 & 0.0131 \\
\hline & & Q2 & -0.023 & 0.002 & -0.812 & & \\
\hline & & Q3 & -0.033 & -0.008 & -1.291 & & \\
\hline & & Q4 & -0.148 & -0.123 & -5.621 & & \\
\hline
\end{tabular}

TABLE 3. The Relative Contribution of ION or EA Lesion Success to the Effect Size Observed in Anisometropia and Vitreous Chamber Depth Asymmetry (T-C)

The regression coefficient $(B)$, standardized effect size at 7 days PS $(b), T$ statistic $(t)$, and the degree to which addition of quartile groups (as defined by either ION $\left[I_{Q 1-4}\right]$ or EA $\left[E A_{Q 1-4}\right]$ lesion success) all contributed to the goodness of fit when compared with a baseline model containing the random effect, Subject ID, and an intercept of $1(\Lambda)$, as defined by the $\chi^{2}$ statistic $\left(\chi^{2}\right)$ and the $P$ value $(P)$. 

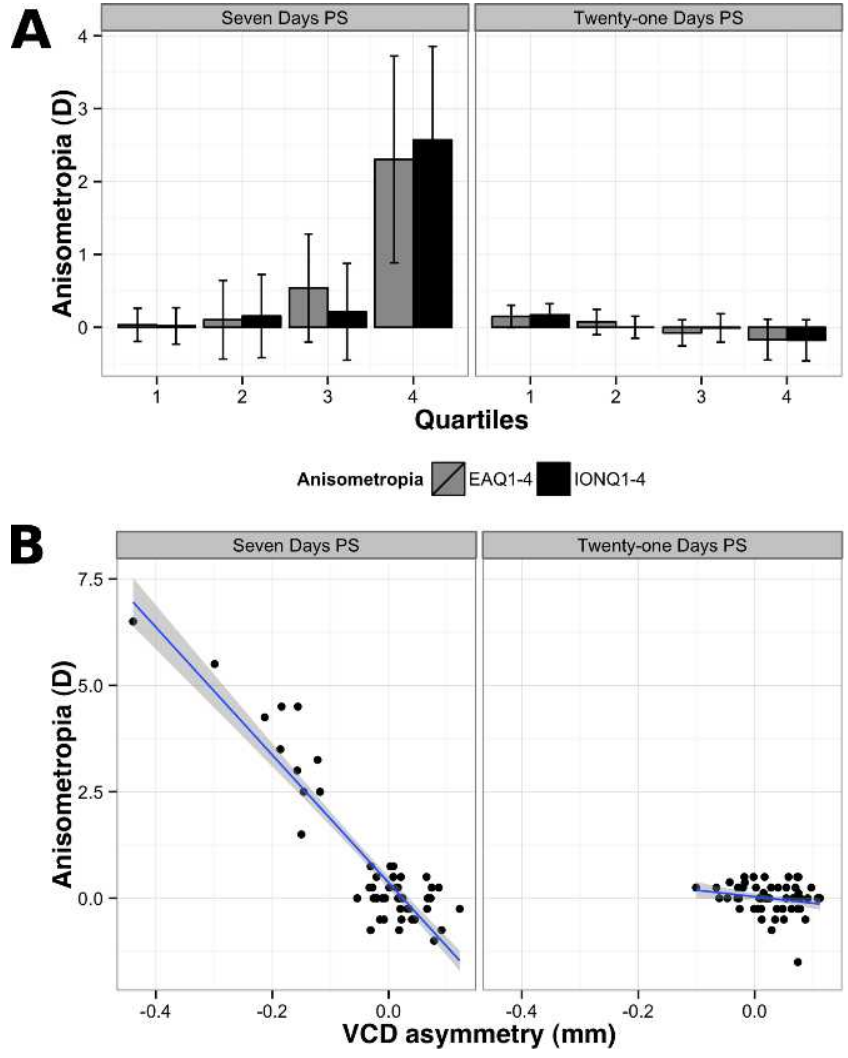

Figure 3. (A) Anisometropia in chicks divided into quartile groups as a function of isthmo-optic nucleus (IONQ1-4, black bars) and ectopic area (EAQ1-4, gray bars) lesion success, at 7 (left facet) and 21 days postsurgery (right facet). (B) Anisometropia plotted against vitreous chamber depth asymmetry $(\triangle V C D) 7$ days (left facet) and 21 days (right facet) postsurgery. Error bars in (A) and the shaded region around the trend lines in (B): 95\% confidence intervals

$\triangle A X L(B=0.173 \pm 0.033, t=5.278)$ measurements was apparent, showing that there was an increase in the values of $\triangle V C D$ over the course of the two measurement time points. In context, this relationship is representative of the initial treatedeye minus control-eye asymmetry being reduced from a negative integer (i.e., the treated eye was relatively shorter than the control eye) at 7 days PS, toward zero (and relative symmetry) at 21 days PS.

\section{Corneal Radius of Curvature}

Comparison of fellow eyes (treated eye minus control eye; $\triangle C R$ ) showed significant corneal flattening of treated eyes relative to control eyes in both the Q3 $(0.035 \pm 0.057 \mathrm{~mm}, d f$ $=12, t=2.222, P=0.046)$ and the Q4 $(0.045 \mathrm{~mm} \pm 0.065, d f=$ $13, t=2.632, P=0.021$ ) groups at 21 days PS (Table 2 ). None of the included categorical fixed effects or the continuous variables was found to exhibit noticeable group specific relationships with the observed variability in $\Delta C R$ (Table 2 , Fig. 4).

\section{ION/EA}

Although, as expected, a strong correlation existed between ION and EA percentage lesion success $(\rho=0.766, d f=52, t=$ 8.604, $P<0.001$; Fig. 1), the high degree of accuracy afforded by the method used to determine lesion success enabled an investigation into whether the lesion effects described above
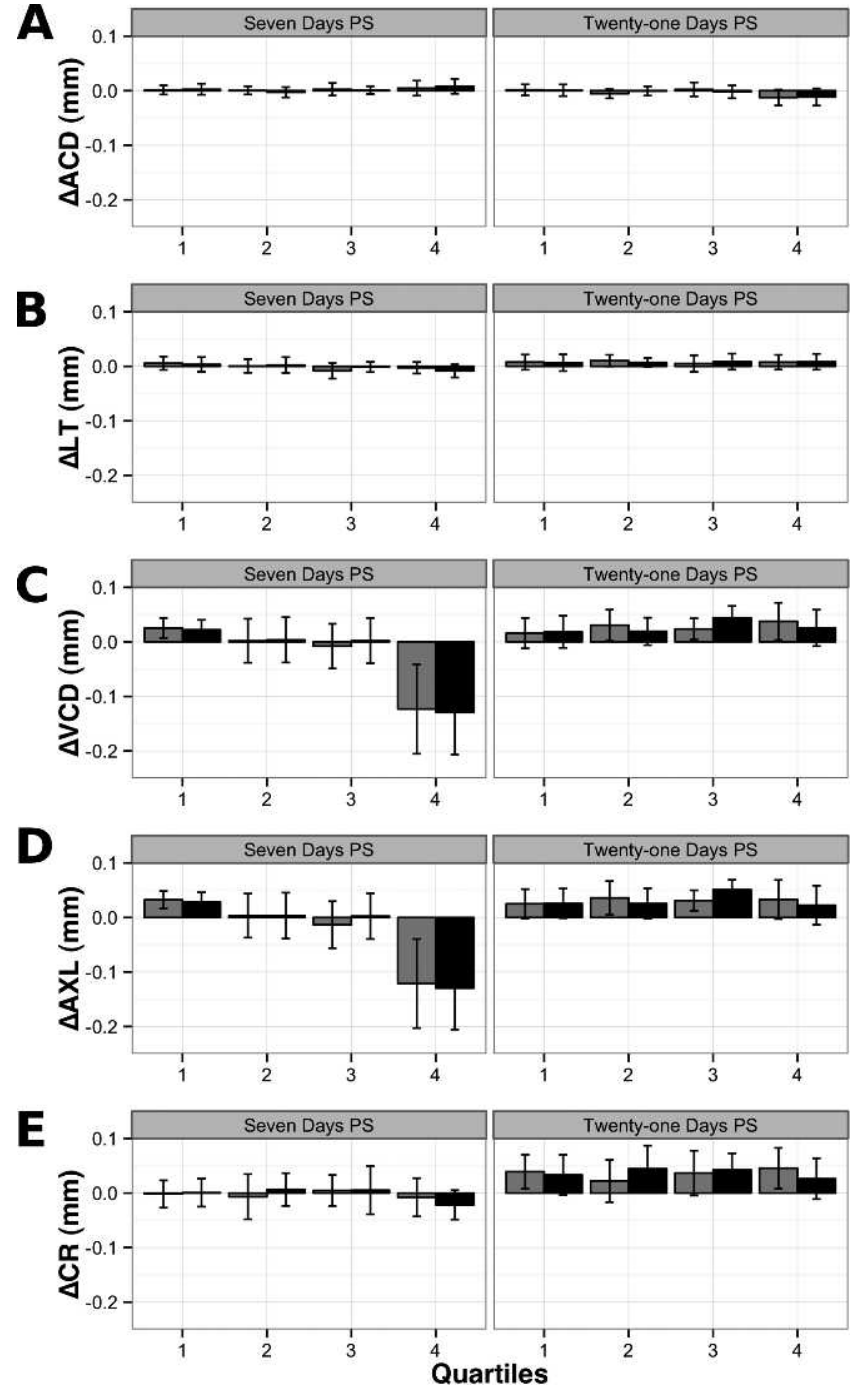

FIGURE 4. (A-E) Ocular component dimension asymmetry in chicks divided into quartile groups as a function of ectopic area (gray bars) and isthmo-optic nucleus (black bars) lesion success, at 7 (left facets) and 21 days PS (right facets). Error bars: $95 \%$ confidence intervals.

arose more as a result of the restricted efferents of the ION or the divergent efferent fibers of the EA.

The strength of association between the variance in both anisometropia and VCD asymmetry, with quartile groups of lesion success defined by the degree of damage to isthmo-optic neurons $\left(\mathrm{ION}_{Q 1-4}\right)$, was greater than that defined by centrifugal EA neuron damage $\left(\mathrm{EA}_{Q 1-4}\right)$ (Table 3 ). In the case of both $\mathrm{ION}_{Q 1-4}$ and $\mathrm{EA}_{Q 1-4}$, the $\mathrm{Q} 4$ group exhibited the strongest relationship (i.e., positive in the case of anisometropia and negative in the case of $\triangle \mathrm{VCD}$ ) with the observed variance. However, addition of the fixed effect, $\mathrm{ION}_{Q 1-4}$, alone to a baseline model generated to explain the variance in anisometropia or $\triangle \mathrm{VCD}$, with an intercept of 1 and containing the random effect Subject ID alone, improved the goodness of fit of the model to a greater degree than did $\mathrm{EA}_{Q 1-4}$ (Table 3).

\section{Discussion}

Our findings indicate that, even under normal visual experience, unilateral disruption of centrifugal ION and EA efferents to the retina of the contralateral eye initially induced a 

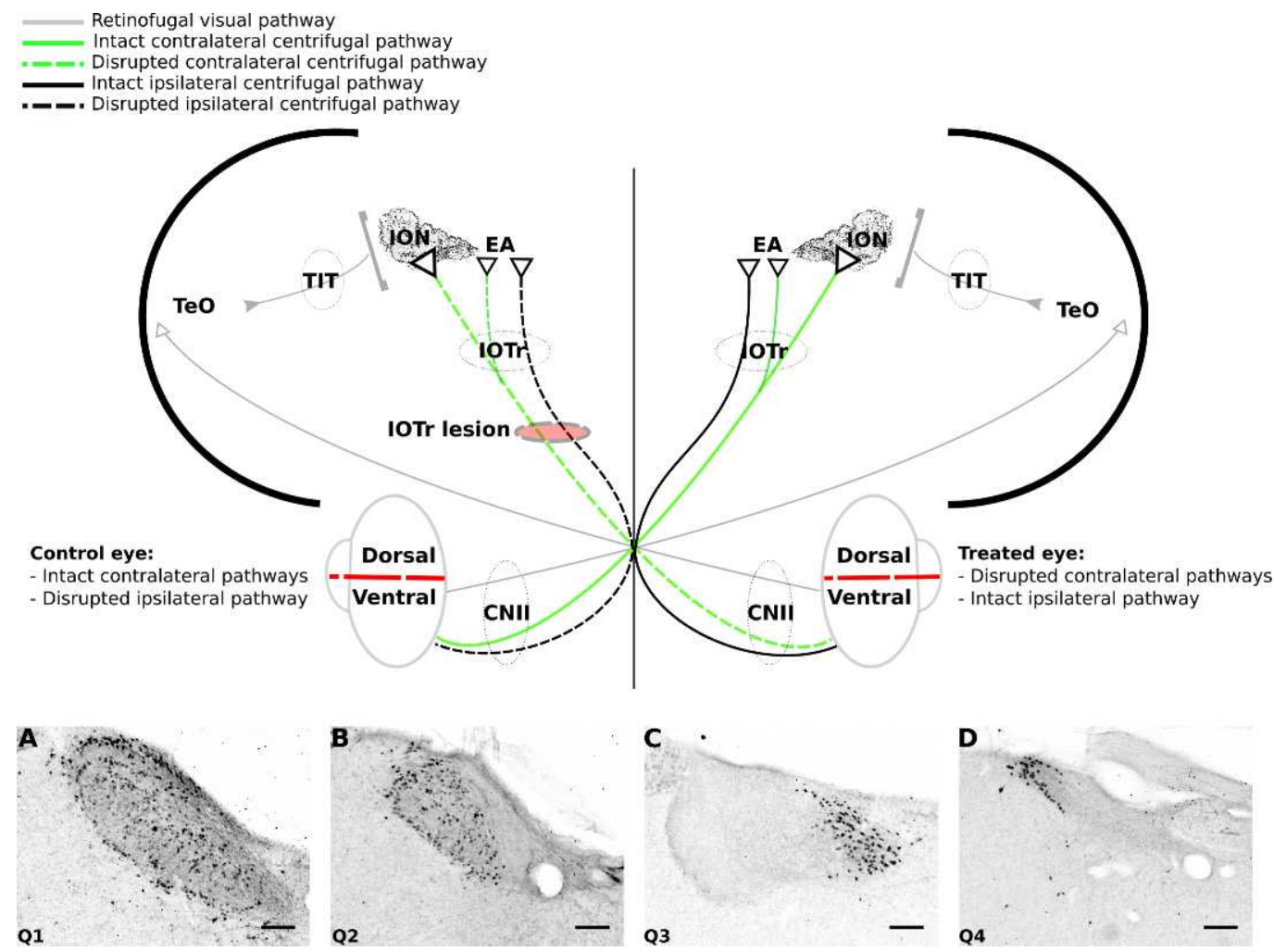

FiguRE 5. A schematic illustration of the main afferent and efferent projections of centrifugal neurons. RGC efferents project to the optic tectum via the optic nerve (CNII). The optic tectum then provides the primary afferent input to the isthmo-optic nucleus (ION) and, potentially, the ectopic area (EA) via the tecto-isthmic tract (TIT). The ION efferents innervate target cells in the contralateral ventral retina. Whereas the majority of EA neurons also project contralaterally, a small number of neurons innervate the ipsilateral retina. In both cases, axon termination is confined to the ventral retina. These centrifugal axons reach their retinal targets via the isthmo-optic tract (IOTr). Unilateral lesion of the IOTr disrupts contralateral ION and EA centrifugal pathways (dashed green pathways), as well as presumably the EA projection to the ipsilateral retina (dashed black pathway). In addition, ipsilateral innervation of the contralateral eye remains intact (solid black pathway). The resulting innervation of treated (contralateral) and control (ipsilateral) eyes is summarized. Photomicrographs (A-D) show examples of retrograde WGA labeling of centrifugal neurons in coronal midbrain sections in chicks from each quartile group of lesion success (Q1-Q4). Scale bars: $150 \mu \mathrm{m}$.

moderate hyperopia at 7 days PS as a result of a reduced vitreous chamber depth in the treated eye (i.e., contralateral to the lesion) relative to that of the control, ipsilateral eye (Fig. 1). Twenty-one days PS, the observed refractive error was largely resolved (Fig. 3). These findings suggest that the diminished accuracy of emmetropization seen in previous work (e.g., in ONS studies) ${ }^{10}$ was at least in part due to the severance of centrifugal axons.

Lesions of both the ION and the IOTr elicit the same end result, that is, the interruption of centrifugal input to the retina through either destruction of ION and EA somata themselves or severance of the axon bundle, respectively. Indeed, the latter in turn would cause retrograde degeneration of centrifugal neurons, albeit over a different time course. Complexities arise, however, when one considers the numerically minor ipsilateral EA projection. The ipsilateral EA tract has been assumed to reach its destination by remaining uncrossed at the optic chiasm. ${ }^{33,45}$ If true, a lesion of either the ION or the IOTr would not only disrupt the bulk of the efferent fibers that travel to the contralateral ("treated") eye but also the small number of efferents that make their way to the ipsilateral ("control") eye. Furthermore, the contralateral treated eye would still receive normal ipsilateral centrifugal EA neuron innervation (Fig. 5).

As a result of this ambiguity in the distinction between treated and control eyes, it is crucial to address the question of whether the induced relative hyperopia in the treated eye was due to the treated eye becoming relatively shorter or the control eye becoming longer than normal. At 7 days PS, a significant positive correlation between the refractive error of treated eyes and combined ION and EA lesion success of individual chicks was evident $(\rho=0.269, P=0.048)$. This, in combination with a weak negative correlation for this relationship in the control eye at the same time point, strongly suggests that the significant anisometropia observed at 7 days PS was due to hyperopia in the treated eyes, whereas the control eyes maintained emmetropia (i.e., a treated eye effect). Furthermore, a significant negative correlation between treated eye refractive error and lesion success at 21 days PS $(\rho=-0.271, P=0.005)$, in combination with a weak negative relationship between control eye refractive error and lesion success, is suggestive of a trend toward an overcompensation for the earlier hyperopia and, thus, relative myopia in treated eyes by the end of the measurement period (Fig. 6).

The finding that relative hyperopia is induced following disruption of a significant proportion of centrifugal neurons (i.e., $>80 \%$ ) is perhaps most logically considered in the context of ONS emmetropization studies, because severance of the optic nerve not only transects retinal efferent axons, but also centrifugal efferents (retinal afferent input from the CVS). Without both brain-retina and retina-brain connections, chicks develop moderate hyperopia. Here, we demonstrate that, following transection of the approximately $0.5 \%$ of the optic nerve fibers that comprise efferent neurons, ${ }^{46}$ a comparable, but transient, response occurs. 


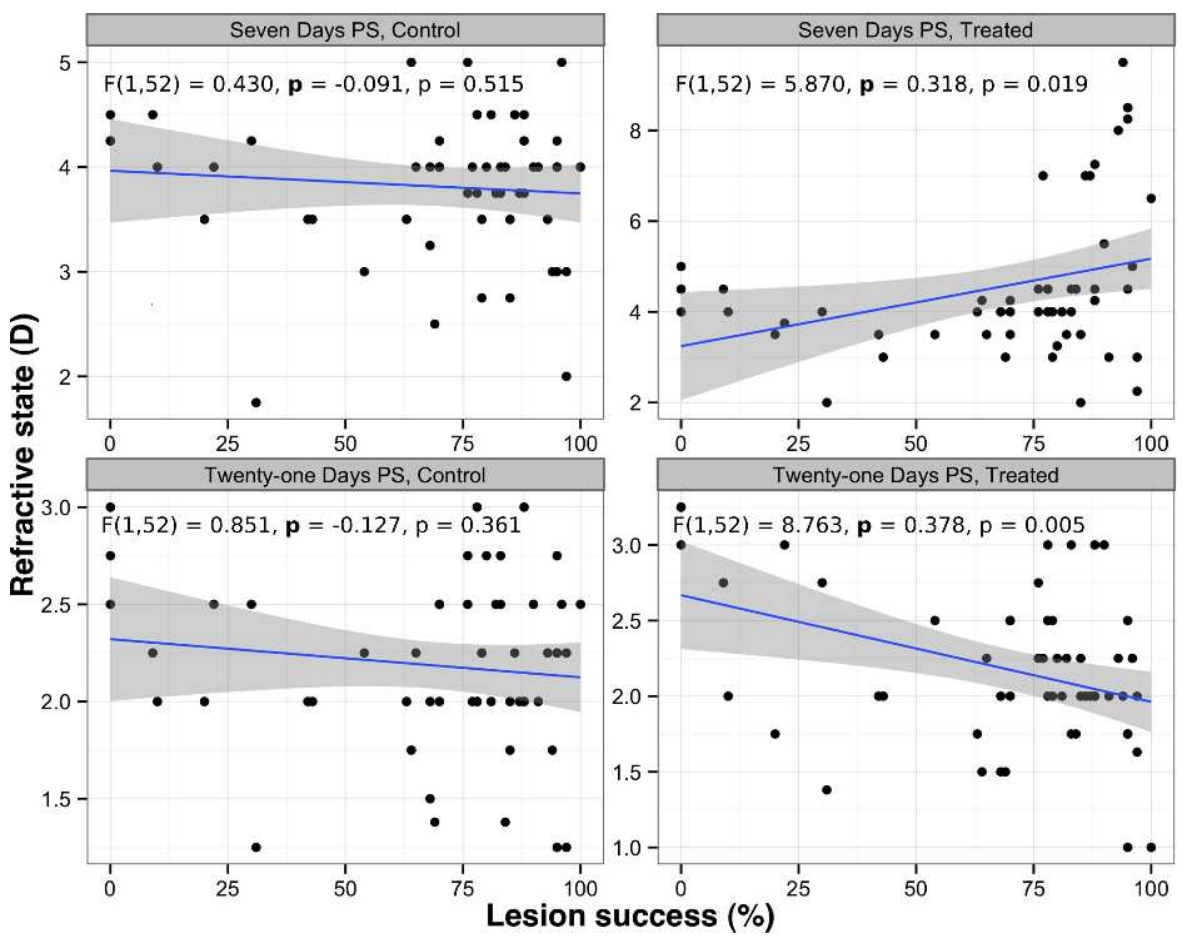

FigurE 6. Refractive error of treated and control eyes at 7 (upper facets) and 21 days (lower facets) PS time points, plotted against percentage lesion success. Seven days PS, a significant positive trend was evident in the treated eye (right facets) but not the control eye (left facets), suggesting that the VCD-dependent anisometropia present at this time point was due to a treated eye effect. Twenty-one days PS, again no significant trend was evident in the control eye. However, a significant, negative trend was found in the treated eye (i.e., evidence toward an apparent overshoot in correction from the ametropia at 7 days PS). Shaded regions around trend lines represent $95 \%$ confidence intervals of variability.

Our findings warrant a reinterpretation of past ONS studies and, specifically, a consideration of the effects of retrograde degeneration of RGCs following axotomy. Hyperopia induced by ONS surgery has been shown to persist for at least 4 weeks, although approximately $75 \%$ of the hyperopia present after 4 weeks had developed after only 1 week. ${ }^{10}$ Two plausible explanations exist for the enduring versus transient difference in the nature of the hyperopia induced by ONS and IOTr lesion, respectively: (1) more than one emmetropization mechanism (i.e., as well as centrifugal modulation) is dependent on both brain-retina and retina-brain connections, such as a systemic (i.e., endocrine response) under light-dependent circadian control; or (2) ONS surgery-induced retrograde degeneration of RGCs that in some way prevents recovery of the hyperopia resulting from loss of the CVS efferents.

The interpretation of our results was complicated by the occurrence of a small systematic error in our measurements of ocular component dimensions, whereby right eye measurements were found to be consistently, albeit marginally, longer than those in the left eye (e.g., AXL; right minus left difference $=$ $0.035 \mathrm{~mm}$ ). One possible explanation is that this observation is the product of an apparatus-based measurement error as we have now, retrospectively, documented its occurrence in a large study involving a different strain of chick (the effect having been missed initially, due to the massively greater effects of form deprivation). ${ }^{47}$ That being said, an alternative explanation is that this observation represents a developmental symptom of normal visual development. If the observed mean asymmetry was the manifestation of developmental asymmetry, based on refractive indices and calculations derived from the White Leghorn strain of chicken, ${ }^{48}$ one would expect a relative elongation of approximately $0.035 \mathrm{~mm}$ in $\triangle A X L$ (Table 2) to induce approximately $0.75 \mathrm{D}$ of relative myopia. Interestingly, however, no refractive asymmetry was observed in our data $(<0.20 \mathrm{D}$ after 21 days PS). Instead, we found that the relative right eye AXL elongation was accompanied by a relative corneal flattening in this eye, equating to a decrease in refractive power of approximately $1.4 \mathrm{D}$, thus, negating much of the AXL asymmetry to within the limits of measurement error from streak retinoscopy, and accounting, to a degree, for the observed refractive symmetry. Crucially, the observed $\triangle A X L$ and $\triangle C R$ were the products of independent measurement instruments (i.e., A-scan ultrasonography and infrared keratometry, respectively). In any event, this small artifact, developmental or otherwise, in the context of the current work, would actually serve to give a conservative account of our findings, since the error acts to bias toward an elongated right eye, whereas here we report a shorter right (treated) eye. On the other hand, this slight bias may account for the small but statistically significant axial length and vitreous chamber depth elongation observed in the Q1 group of chicks after 7 days postsurgery and also in the Q2, Q3, and Q4 group asymmetry after 21 days PS, that all fall within or around the limits of the bias described.

Although it is plausible that other retina-brain mechanisms regulate emmetropization, it may be the case that the severe hyperopia reported following ONS surgery is largely due to the effects of retrograde degeneration of RGCs (Choh V, et al. IOVS 2004; 45:ARVO E-Abstract 47). ${ }^{10,13}$ If modulation of eye growth by centrifugal efferents constitutes the only extraretinal influence on emmetropization, our findings represent the actual, albeit less severe, effects of brain-retina disconnectivity. As mentioned, it has been suggested that the effect observed in ONS studies could be the result of retinal ganglion cell (RGC) death. However, Troilo et al. ${ }^{10}$ noted that, prior to the onset of RGC degeneration (i.e., 7 days PS), ${ }^{49}$ significant hyperopia was already apparent. Furthermore, if the moderate hyperopia observed in our results were due to the degeneration of centrifugal target cells, recovery from the initial ametropia 
presumably would not take place. An alternative view is that, rather than the target cells of centrifugal efferents becoming progressively functionally quiescent, deafferentation may have induced a short period, (i.e., 5-7 days) of hyperactivity in IOTCs, which induced the ocular growth changes reported in this study (i.e., an epileptogenic cell response). ${ }^{50,51}$ However, once centrifugal target cell degeneration had taken place, this hyperderegulation of intraretinal mechanisms would presumably no longer occur, thus corresponding to the beginning of the recovery period observed here.

Typically, lesion success is quantified through volumetric estimations from Nissl-stained tissue ${ }^{52}$ or from coincidental behavioral deficits that result from a given lesion site, such as the absence of pupillary constriction response following lesion of the optic nerve. ${ }^{53}$ However, our pathway-tracing paradigm (i.e., retrograde labeling residual intact centrifugal neurons through intravitreal injection of WGA in the eye contralateral to the lesioned side of the midbrain) allowed for a greater accuracy in the determination of the number, location, and type (i.e., ION or EA neuron) of the remaining intact centrifugal connections than would a typical histologic analysis of the lesion site from Nissl-stained tissue. A complete lesion of the IOTr would, in theory, prevent all retrograde transmission of WGA to the ION/ EA. However, we found that even an apparently complete lesion of the IOTr (as assessed by Nissl staining) rarely abolished WGA retrograde labeling within the ION/EA complex entirely. Although the reasons for this are unknown, several logical possibilities exist: (1) Not all efferent axons take the same route to the retina; (2) the position of a given lesion was caudal to where the axons of the intact connections joined the fiber bundle; (3) as a result of the lesion technique, portions of the myelinated axons were spared; and (4) given the age of the chicks used, and therefore the high degree of neuronal plasticity, recovery mechanisms, such as regenerative growth or compensatory branching of intact fibers, may have occurred during the experimental time frame. ${ }^{51,54}$

Indeed, the activity/behavior of neurons following partial or full deafferentation is not fully understood. In 1969, von Monakow proposed that, whereas the local effect of a lesion is straightforward (i.e., removal of neural elements), the effect of a lesion at a distance on neurons to which the area of damage was connected is more complex, inducing "diaschisis," 55,56 whereby partially deafferented neurons can initially lose all responsiveness to any intact input for a given period and, subsequently, reestablish function after the initial "shock." Further, diaschisis may be followed by "denervation supersensitivity," a phenomenon described in the peripheral nervous system, whereby deafferentation of neurons induces a heightened state of reactivity to subsequent experimentally induced input..$^{57,58}$ In the case of partial deafferentiation, the affected neuron would become more responsive to the summation of the remaining input from intact afferents. In a study aimed at elucidating retinal connectivity of centrifugal efferents, Nickla et al. ${ }^{59}$ found that cytochrome oxidase labeling of IOTCs was severely diminished ( $\sim 97 \%)$ as a result of IOTr lesion. Survival times used in the study by Nickla and colleagues were between 9 and 36 days PS, and it was noted that the severity of the reduction in cytochrome oxidase labeling increased with the length of survival time. This suggests that the process of deafferentation-induced functional quiescence in IOTCs was progressive over a duration comparable to that used in our study. Because the earliest time point (i.e., 9 days PS) was beyond the time point within which treated eye hyperopia developed in our study (i.e., 7 days PS), IOTr lesion induced changes in cytochrome oxidase labeling at time points corresponding to the lesion effect observed in our study cannot be determined. Interestingly, however, the suggested time frame during which one would expect to observe the physiologic side effects of diaschisis (1 week or less) and subsequent denervation supersensitivity (7-28 days PS) are well matched to the pattern of ocular growth effects reported here.

A strong positive correlation existed between percentage ION and EA lesion success as a result of their common axonal pathway taken to the retina (i.e., the IOTr). However, following analysis of the lesion effect on ocular phenotype as a function of percentage ION and EA lesion success independently, it was evident that percentage ION lesion success was a stronger predictor than was percentage EA lesion success, of the observed variance in the dependent variables anisometropia and $\triangle \mathrm{VCD}$. Our recent pathway-tracing experiments (in preparation) suggest that, unlike the ION, EA neurons do not receive direct tectal afferents (i.e., visual input from the tectofugal pathway). Taken together with the results reported here, it is possible that the role of centrifugal efferents in emmetropization is likely to be the result of the highly topographic connectivity of the "closed loop" ION-retina-optic tectum-retina circuitry, ${ }^{60}$ characterized by restricted efferent termination within the retina rather than from the potentially visually independent, widespread efferent termination of EA neurons. ${ }^{36}$

Intravitreal injection of the nonspecific nitric oxide synthase inhibitor L-NAME has been shown to inhibit the increase in choroidal thickness associated with the normal compensatory response to myopic defocus in the chick. ${ }^{40}$ The retinal target cells of centrifugal efferents (IOTCs) synthesize the gaseous signal molecule nitric oxide. ${ }^{34,38,61,62}$ Thus, the hyperopic shift observed in the contralateral eyes of ION/IOTr lesioned chicks reported in our study could have resulted from the VCD-dependent changes that arise from nitric oxide release-induced choroidal thickening. Indeed, choroidal thickening has been reported following ONS surgery. ${ }^{13}$ However, in the absence of choroidal thickness data from chicks in our study, we are unable to substantiate this possibility. Intriguingly, however, it has been reported in rats that, following ONS surgery, RGC death takes place via a mechanism of nitric oxide-mediated excitotoxicity. ${ }^{63}$ Expression of NOS within RGCs peaked at 5 days PS and had then decreased to near normal levels by 28 days postsurgery. If this sequence of events also occurs in chicks, such a response could explain the transient nature of the hyperopic shift observed following ION and IOTr lesion surgeries. Alternatively, compensatory events at the lesion site itself could also explain the transient nature of the lesion effect described above: (1) the "recovery of function" phenomenon (i.e. "rewiring" at the lesion site resulting in a restoration of function) ${ }^{64}$ or (2) compensatory hyperactivity of neighboring surviving centrifugal neurons.

Finally, having considered our findings predominantly in the context of modulation of intraocular emmetropization mechanisms by higher brain centers, and in particular, numerous brain-retina disconnectivity studies, it is interesting to note the relevance of our findings to the ongoing attempts to elucidate the function, or functions, of the CVS. Over more than a century, numerous hypotheses relating to CVS function have been put forward based on anatomic, ${ }^{36,37,65}$ physiologic, ${ }^{66}$ and behavioral evidence. ${ }^{28,67}$ According to the premise of the principle of proper mass, an increased dependence on a given functional modality is positively correlated with the relative size of the neural structure, or structures, upon which it is dependent. ${ }^{68}$ Gutierrez-Ibanez et al. ${ }^{19}$ have reported that increased CVS complexity (as defined by ION lamination, volume, and number of cells) was positively correlated with the presence of lower field myopia. This is an optical specialization predominantly found in ground-feeding bird species $^{69,70}$ (including chickens and some amphibians ${ }^{71}$ ), which allows the ground, horizon, and sky to be kept in focus at the same time. ${ }^{19,70}$ Thus, our study and that of Gutierrez-Ibanez et al. ${ }^{19}$ both implicate the CVS in ocular growth mechanisms. 
Taken together, these findings raise the possibility of a regional effect in the present study, with perhaps a greater hyperopic shift/growth inhibition being present in the ventral retina, where the centrifugal target cells are most prevalent.

The fact remains that centrifugal efferents are "functional" in adult birds (i.e., they are known to modulate retinal ganglion cell activity) ${ }^{60}$ and, during development, the CVS undergoes a period of extensive neuronal pruning involving a reduction of nearly $60 \%$ in cell number. ${ }^{21}$ Therefore, it seems unlikely that the CVS functions solely to modulate intraocular emmetropization mechanisms during the early posthatch critical period. Gutierrez-Ibanez et al. ${ }^{19}$ suggest that, in certain species, the CVS is involved in the development of lower field myopia and, in adulthood, acts functionally to switch visual attention between an emmetropic upper visual field and a myopic lower field. The fact that disruption of CVS can result in changes in refractive development, as reported here, provides at least some limited support for this theory.

In conclusion, our findings show that, in the absence of centrifugal innervation of the retina, early refractive and ocular development of the chick is transiently disrupted. Although it has previously been demonstrated that intact brain-retina connectivity is not required for active compensation to imposed defocus, resultant deficits have been found in its absence (e.g., hyperopia following ONS). We report here a comparable initial hyperopia following electrolytic lesion of the IOTr/ION, which is consistent with the response to ONS, and propose that ONS-induced hyperopia is, at least in part, due to the severing of centrifugal efferents.

\section{Acknowledgments}

The authors thank William Hodos and Wayne Kuenzel for their intellectual contributions and Rob Thomas for statistical advice.

A portion of this work has been published previously as an ARVO abstract: Dillingham CM, et al. IOVS 2012;53:ARVO E-Abstract 3432.

Supported by Biotechnology and Biological Sciences Research Council Grant BB/D526145/1.

Disclosure: C.M. Dillingham, None; J.A. Guggenheim, None; J.T. Erichsen, None

\section{References}

1. Wallman J, Adams JI. Developmental aspects of experimental myopia in chicks: susceptibility, recovery and relation to emmetropization. Vision Res. 1987;27:1139-1163.

2. Schaeffel F, Howland HC. Properties of the feedback loops controlling eye growth and refractive state in the chicken. Vision Res. 1991;31:717-734.

3. Troilo D, Wallman J. The regulation of eye growth and refractive state: an experimental study of emmetropization. Vision Res. 1991;31:1237-1250.

4. Wildsoet C, Wallman J. Choroidal and scleral mechanisms of compensation for spectacle lenses in chicks. Vision Res. 1995; 35:1175-1194.

5. Chen YP, Prashar A, Erichsen JT, To CH, Hocking PM, Guggenheim JA. Heritability of ocular component dimensions in chickens: genetic variants controlling susceptibility to experimentally induced myopia and pretreatment eye size are distinct. Invest Ophthalmol Vis Sci. 2011;52:4012-4020.

6. Wallman J, Adams JI, Trachtman JN. The eyes of young chickens grow toward emmetropia. Invest Ophthalmol Vis Sci. 1981;20:557-561.

7. Kee CS, Marzani D, Wallman J. Differences in time course and visual requirements of ocular responses to lenses and diffusers. Invest Ophthalmol Vis Sci. 2001;42:575-583.
8. Mertz JR, Wallman J. Choroidal retinoic acid synthesis: a possible mediator between refractive error and compensatory eye growth. Exp Eye Res. 2000;70:519-527.

9. Irving EL, Sivak JG, Callender MG. Refractive plasticity of the developing chick eye. Ophthalmic Physiol Opt. 1992;12:448-456.

10. Troilo D, Gottlieb MD, Wallman J. Visual deprivation causes myopia in chicks with optic nerve section. Curr Eye Res. 1987;6:993-999.

11. McBrien NA, Moghaddam HO, Cottriall CL, Leech EM, Cornell LM. The effects of blockade of retinal cell action potentials on ocular growth, emmetropization and form deprivation myopia in young chicks. Vision Res. 1995;35:1141-1152.

12. Choh V, Padmanabhan V, Li WS, Sullivan AB, Wildsoet CF. Colchicine attenuates compensation to negative but not to positive lenses in young chicks. Exp Eye Res. 2008;86:260-270.

13. Wildsoet C. Neural pathways subserving negative lens-induced emmetropization in chicks-insights from selective lesions of the optic nerve and ciliary nerve. Curr Eye Res. 2003;27:371-385.

14. Wallman J, Gottlieb MD, Rajaram V, Fugate-Wentzek LA. Local retinal regions control local eye growth and myopia. Science. 1987; 237:73-77.

15. Miles FA, Wallman J. Local ocular compensation for imposed local refractive error. Vision Res. 1990;30:339-349.

16. Schaeffel F, Troilo D, Wallman J, Howland HC. Developing eyes that lack accommodation grow to compensate for imposed defocus. Vis Neurosci. 1990;4:177-183.

17. Wildsoet CF, Schmid KL. Optical correction of form deprivation myopia inhibits refractive recovery in chick eyes with intact or sectioned optic nerves. Vision Res. 2000;40:32733282.

18. Reperant J, Ward R, Miceli D, et al. The centrifugal visual system of vertebrates: a comparative analysis of its functional anatomical organization. Brain Res Rev. 2006;52:1-57.

19. Gutierrez-Ibanez C, Iwaniuk AN, Lisney TJ, Faunes M, Marin GJ, Wylie DR. Functional implications of species differences in the size and morphology of the isthmo optic nucleus (ION) in birds. PLoS One. 2012;7:e37816.

20. Wilson M, Lindstrom SH. What the bird's brain tells the bird's eye: the function of descending input to the avian retina. Vis Neurosci. 2011;28:337-350.

21. Clarke PG, Rogers LA, Cowan WM. The time of origin and the pattern of survival of neurons in the isthmo-optic nucleus of the chick. J Comp Neurol. 1976;167:125-142.

22. Marin G, Letelier JC, Wallman J. Saccade-related responses of centrifugal neurons projecting to the chicken retina. Exp Brain Res. 1990;82:263-270.

23. Miles FA. Centrifugal control of the avian retina. 3. Effects of electrical stimulation of the isthmo-optic tract on the receptive field properties of retinal ganglion cells. Brain Res. 1972;48: 115-129.

24. Woodson W, Reiner A, Anderson K, Karten HJ. Distribution, laminar location, and morphology of tectal neurons projecting to the isthmo-optic nucleus and the nucleus isthmi, pars parvocellularis in the pigeon (Columba livia) and chick (Gallus domesticus): a retrograde labelling study. J Comp Neurol. 1991;305:470-488.

25. Cowan WM, Powell TP. Centrifugal fibres to the retina in the pigeon. Nature. 1962;194:487.

26. Galifret Y, Condé-Courtine F, Repérant J, Servière J. Centrifugal control in the visual system of the pigeon. Vision Res. 1971; 3(suppl):185-200.

27. Uchiyama $\mathrm{H}$, Ito $\mathrm{H}$. Target-cells for the isthmo-optic fibers in the retina of the Japanese quail. Neurosci Lett. 1993;154:35-38.

28. Uchiyama H, Ohno H, Kodama R. Lesion of the isthmo-optic nucleus impairs target selection for visually guided reaching. Behav Brain Res. 2012;233:359-366. 
29. Miceli D, Repérant J, Bertrand C, Rio JP. Functional anatomy of the avian centrifugal visual system. Behav Brain Res. 1999;98: 203-210.

30. McGill JI, Powell TP, Cowan WM. Retinal representation upon optic tectum and isthmo-optic nucleus in pigeon. $J$ Anat. 1966;100:5-33.

31. Crossland WJ, Hughes CP. Observations on the afferent and efferent connections of the avian isthmo-optic nucleus. Brain Res. 1978;145:239-256.

32. Cowan WM, Adamson L, Powell TP. An experimental study of the avian visual system. J Anat. 1961;95:545-563.

33. Clarke PG, Cowan WM. Ectopic neurons and aberrant connections during neural development. Proc Natl Acad Sci U S A. 1975;72:4455-4458.

34. Lindstrom SH, Nacsa N, Blankenship T, et al. Distribution and structure of efferent synapses in the chicken retina. Vis Neurosci. 2009;26:215-226.

35. Maturana HR, Frenk S. Synaptic connections of the centrifugal fibers in the pigeon retina. Science. 1965;150:359-361.

36. Woodson W, Shimizu T, Wild JM, Schimke J, Cox K, Karten HJ. Centrifugal projections upon the retina-an anterograde tracing study in the pigeon (Columba livia). J Comp Neurol. 1995;362:489-509.

37. Catsicas S, Catsicas M, Clarke PG. Long-distance intraretinal connections in birds. Nature. 1987;326:186-187.

38. Morgan IG, Miethke P, Li ZK. Is nitric oxide a transmitter of the centrifugal projection to the avian retina. Neurosci Lett. 1994; 168:5-7.

39. Fujikado T, Kawasaki Y, Fujii J, et al. The effect of nitric oxide synthase inhibitor on form-deprivation myopia. Curr Eye Res. 1997; 16:992-996.

40. Nickla DL, Wilken E, Lytle G, Yom S, Mertz J. Inhibiting the transient choroidal thickening response using the nitric oxide synthase inhibitor L-NAME prevents the ameliorative effects of visual experience on ocular growth in two different visual paradigms. Exp Eye Res. 2006;83:456-464.

41. Kuenzel WJ, Masson M. A Stereotaxic Atlas of the Brain of the Chick (Gallus domesticus). Baltimore: Johns Hopkins University Press; 1988.

42. Prashar A, Hocking PM, Erichsen JT, Fan Q, Saw SM, Guggenheim JA. Common determinants of body size and eye size in chickens from an advanced intercross line. Exp Eye Res. 2009;89:42-48.

43. Schaeffel F, Howland HC. Corneal accommodation in chick and pigeon. J Comp Physiol A. 1987;160:375-384.

44. Akaike H. A new look at the statistical model identification. IEEE Trans Automat Control. 1974;19:716-723.

45. O'Leary DD, Cowan WM. Further studies on the development of the isthmo-optic nucleus with special reference to the occurrence and fate of ectopic and ipsilaterally projecting neurons. J Comp Neurol. 1982;212:399-416.

46. Rager G, Rager U. Systems-matching by degeneration. 1 . Quantitative electron-microscopic study of generation and degeneration of retinal ganglion cells in chicken. Exp Brain Res. 1978;33:65-78.

47. Chen YP, Hocking PM, Wang L, et al. Selective breeding for susceptibility to myopia reveals a gene-environment interaction. Invest Ophthalmol Vis Sci. 2011;52:4003-4011.

48. Avila NV, McFadden SA. A detailed paraxial schematic eye for the White Leghorn chick. J Comp Physiol A Neuroethol Sens Neural Behav Physiol. 2010;196:825-840.

49. Muchnick N, Hibbard E. Avian retinal ganglion cells resistant to degeneration after optic nerve lesion. Exp Neurol. 1980;68: 205-216.

50. Goddard GV, McIntyre DC, Leech CK. A permanent change in brain function resulting from daily electrical stimulation. Exp Neurol. 1969;25:295-330.
51. O'Keefe J, Nadel L. Introduction to the Lesion Review. The Hippocampus as a Cognitive Map. Oxford, UK: Oxford University Press; 1978:231-239.

52. Knipling RR. No deficit in near-field visual acuity of pigeons after transection of the isthmo-optic tract. Physiol Behav. 1978;21:813-816.

53. Li T, Howland HC. Modulation of constant light effects on the eye by ciliary ganglionectomy and optic nerve section. Vision Res. 2000;40:2249-2256.

54. Raisman G. Neuronal plasticity in the septal nuclei of the adult rat. Brain Res. 1969;14:25-48.

55. Schoenfeld TA, Hamilton LW. Secondary brain changes following lesions: a new paradigm for lesion experimentation. Physiol Behav. 1977;18:951-967.

56. von Monakow C. Diaschisis. In: Pribram R, ed. Mood, States, and Mind. Balitmore:Penguin; 1969:68-94.

57. Stavraky GW. Effects of partial denervation on spinal neurones and their possible relation to parkinsonism. Rev Can Biol. 1961;20:159-166.

58. Sharpless SK. Reorganization of function in the nervous system: use and disuse. Annu Rev Physiol. 1964;26:357-388.

59. Nickla DL, Gottlieb MD, Marin G, Rojas X, Britto LR, Wallman J. The retinal targets of centrifugal neurons and the retinal neurons projecting to the accessory optic system. Vis Neurosci. 1994;11:401-409.

60. Li JL, Xiao Q, Fu YX, Wang SR. Centrifugal innervation modulates visual activity of tectal cells in pigeons. Vis Neurosci. 1998;15:411-415.

61. Wilson M, Nacsa N, Hart NS, Weller C, Vaney DI. Regional distribution of nitrergic neurons in the inner retina of the chicken. Vis Neurosci. 2011;28:205-220.

62. Fischer AJ, Stell WK. Nitric oxide synthase-containing cells in the retina, pigmented epithelium, choroid, and sclera of the chick eye. J Comp Neurol. 1999;405:1-14.

63. Lee EJ, Kim KY, Gu TH, et al. Neuronal nitric oxide synthase is expressed in the axotomized ganglion cells of the rat retina. Brain Res. 2003;986:174-180.

64. Stein DG, Rosen JJ, Graziadei J, Mishkin D, Brink JJ. Central nervous system: recovery of function. Science. 1969;166:528530.

65. Holden AL. Centrifugal pathways to the retina: which way does the "searchlight" point? Vis Neurosci. 1990;4:493-495.

66. Uchiyama H, Nakamura S, Imazono T. Long-range competition among the neurons projecting centrifugally to the quail retina. Vis Neurosci. 1998;15:417-423.

67. Rogers LJ, Miles FA. Centrifugal control of the avian retina. V. Effects of lesions of the isthmo-optic nucleus on visual behaviour. Brain Res. 1972;48:147-156.

68. Jerison HJ. Evolution of the Brain and Intelligence. New York: Academic Press; 1973.

69. Murphy CJ, Howland M, Howland HC. Raptors lack lower-field myopia. Vision Res. 1995;35:1153-1155.

70. Hodos W, Erichsen JT. Lower-field myopia in birds: an adaptation that keeps the ground in focus. Vision Res. 1990; 30:653-657.

71. Schaeffel F, Hagel G, Eikermann J, Collett T. Lower-field myopia and astigmatism in amphibians and chickens. J Opt Soc Am A. 1994;11:487-495.

72. Choh V, Lew MY, Nadel MW, Wildsoet CF. Effects of interchanging hyperopic defocus and form deprivation stimuli in normal and optic nerve-sectioned chicks. Vision Res. 2006; 46:1070-1079.

73. Fischer AJ, Morgan IG, Stell WK. Colchicine causes excessive ocular growth and myopia in chicks. Vision Res. 1999;39:685697. 